\title{
Excited state dynamics of liquid water: Insight from the dissociation reaction following two-photon excitation
}

\author{
Christopher G. Elles, llya A. Shkrob, and Robert A. Crowell ${ }^{a)}$ \\ Chemistry Division, Argonne National Laboratory, Argonne, Illinois 60439 \\ Stephen E. Bradforth ${ }^{\text {b) }}$ \\ Department of Chemistry, University of Southern California, Los Angeles, California 90089
}

(Received 11 January 2007; accepted 19 March 2007; published online 25 April 2007)

\begin{abstract}
The authors use transient absorption spectroscopy to monitor the ionization and dissociation products following two-photon excitation of pure liquid water. The primary decay mechanism changes from dissociation at an excitation energy of $8.3 \mathrm{eV}$ to ionization at $12.4 \mathrm{eV}$. The two channels occur with similar yield for an excitation energy of $9.3 \mathrm{eV}$. For the lowest excitation energy, the transient absorption at $267 \mathrm{~nm}$ probes the geminate recombination kinetics of the $\mathrm{H}$ and $\mathrm{OH}$ fragments, providing a window on the dissociation dynamics. Modeling the $\mathrm{OH}$ geminate recombination indicates that the dissociating $\mathrm{H}$ atoms have enough kinetic energy to escape the solvent cage and one or two additional solvent shells. The average initial separation of $\mathrm{H}$ and $\mathrm{OH}$ fragments is $0.7 \pm 0.2 \mathrm{~nm}$. Our observation suggests that the hydrogen bonding environment does not prevent direct dissociation of an $\mathrm{O}-\mathrm{H}$ bond in the excited state. We discuss the implications of our measurement for the excited state dynamics of liquid water and explore the role of those dynamics in the ionization mechanism at low excitation energies. (C) 2007 American Institute of Physics. [DOI: 10.1063/1.2727468]
\end{abstract}

\section{INTRODUCTION}

The excited state dynamics of liquid water plays an important role in a wide range of applications. A prominent example is the irradiation of aqueous systems, where ionized and electronically excited water molecules decompose into reactive species that are responsible for much of the subsequent chemistry. ${ }^{1}$ Although much is known about the kinetics of the transient species, the initial dynamics that governs their formation have not been fully characterized. In this paper we examine the photodissociation of liquid water and consider the role of excited state dynamics in the liquidphase ionization channel.

The isolated water molecule is a useful reference for studying the liquid. The maximum of the first absorption band is near $7.5 \mathrm{eV}$ in the gas phase, corresponding to a transition that promotes an electron from the highest occupied nonbonding valence orbital $\left(1 b_{1}\right)$ to an unoccupied antibonding orbital $\left(4 a_{1} / 3 s\right)$ with a significant Rydberg character. This first electronic excited state, which is well below the $12.6 \mathrm{eV}$ ionization potential of the isolated molecule, is strongly repulsive and promptly dissociates to $\mathrm{H}$ and $\mathrm{OH}$ with a large fraction of the available energy going into relative translation of the fragments. ${ }^{2,3}$ The second excited state corresponds to a transition from the next highest valence orbital $\left(4 a_{1} / 3 s \leftarrow 3 a_{1}\right)$ and also dissociates along an $\mathrm{O}-\mathrm{H}$ bond. Much less is known about the excited state dynamics of liquid water, although a shift of the first absorption band by about $0.7 \mathrm{eV}$ to higher energy indicates that the liquid environment has a strong influence on the electronic structure. $^{4}$

A particularly intriguing aspect of the dynamics of liquid water is the role that hydrogen bonding plays. Hydrogen bonding gives water many of its unusual properties and recent work using multidimensional vibrational spectroscopy, among other techniques, has uncovered many interesting aspects of how hydrogen bonding affects the ground state dynamics. $^{5-7}$ There is far less work addressing the role of cooperative effects on the excited state dynamics of the liquid. ${ }^{8,9}$ Furthermore, because it is difficult to accurately include cooperative effects in electronic structure calculations, only a limited number of theoretical treatments concerning the electronically excited states of condensed-phase water are available. ${ }^{10-15}$ Even fewer calculations explore excited state potential energy surfaces outside of the FranckCondon region, and these are generally limited to the dimer and other small water clusters. ${ }^{16-21}$ Experimental measurements of the dynamics in the bulk liquid provide important benchmarks for comparison with theoretical and computational results.

Previous investigations of the dynamics following electronic excitation of liquid water generally focus on the mechanism of ionization, rather than dissociation. ${ }^{22-26}$ However, early studies irradiating water with vacuum ultraviolet light indicate that both processes play a role in the liquid. ${ }^{27-30}$

$$
\mathrm{H}_{2} \mathrm{O}_{(l)}+h \nu \stackrel{+\mathrm{H}_{2} \mathrm{O}}{\longrightarrow} \mathrm{H}_{3} \mathrm{O}_{(\mathrm{aq})}^{+}+\mathrm{OH}_{(\mathrm{aq})}+e_{(\mathrm{aq})}^{-},
$$




$$
\mathrm{H}_{2} \mathrm{O}_{(l)}+h \nu \rightarrow \mathrm{H}_{(\mathrm{aq})}+\mathrm{OH}_{(\mathrm{aq})} .
$$

Unlike in the gas phase, ionization occurs down to the onset of optical absorption near $6.5 \mathrm{eV}$ for both one- and twophoton excitations of liquid water. ${ }^{30,31}$ The energy for vertical ionization of the liquid is about $11 \mathrm{eV}$, and the discrepancy implies that nuclear motion must play a role in the ionization mechanism at low energies. ${ }^{26}$ The precise relationship of dissociation and ionization, whether they are mutually exclusive pathways or slightly different outcomes of a similar process, ${ }^{32}$ is an unresolved distinction that lies at the heart of understanding the ionization mechanism in this energy regime. Our experiments continue to unravel these details by explicitly examining the excited state dynamics of liquid water.

The work described in this paper uses transient absorption spectroscopy to monitor the products of ionization and dissociation. The dissociation channel plays the more important role for an excitation energy of $8.3 \mathrm{eV}$, while dissociation and ionization occur with roughly equal probability for $9.3 \mathrm{eV}$ excitation, and ionization dominates at $12.4 \mathrm{eV}$. Our result agrees with previous observations that the ionization yield increases rapidly across this range of energies, ${ }^{31,32}$ presumably with a corresponding decrease in the dissociation yield. ${ }^{33}$ At the lower excitation energy, where the dissociation channel dominates, we monitor the geminate recombination kinetics of the dissociation products and determine that the average initial separation of $\mathrm{H}$ and $\mathrm{OH}$ fragments is about $0.7 \mathrm{~nm}$, similar to the value from recent molecular dynamics simulations of dissociating $\mathrm{H}_{2} \mathrm{O}$ in ice. ${ }^{34,35}$ The relatively large separation of the dissociation products suggests that $\mathrm{H}$ atoms are formed with enough kinetic energy to escape the solvent cage and one or two additional solvent shells.

\section{EXPERIMENT}

Time-resolved transient absorption measurements monitor the evolution of the ionization and dissociation products following the two-photon excitation of liquid water. We probe the transient products at two wavelengths: 650 and $267 \mathrm{~nm}$. The solvated electron is the only species that absorbs visible light, and therefore the transient signal that we measure at $650 \mathrm{~nm}$ reflects purely the geminate recombination kinetics following ionization. In contrast, the signal at $267 \mathrm{~nm}$ potentially includes contributions from the kinetics following dissociation as well as ionization, because both electrons and $\mathrm{OH}$ radicals absorb at that wavelength. Hydrogen atoms and hydronium ions do not absorb light at either wavelength.

In addition to the measurements for pure water, we observe the transient change in absorption for a $2 M$ solution of perchloric acid, where protons from the acid rapidly react with solvated electrons from the ionization of water. By eliminating solvated electrons, the measurements in acid solution reveal the relative contribution of $\mathrm{OH}$ radicals to the ultraviolet absorption signal. Comparing the transient $a b-$ sorption for pure water and a $2 \mathrm{M}$ solution of $\mathrm{NaClO}_{4}$ confirms that irradiation of perchlorate ions does not contribute to the signal because the transient absorption is the same in the two solutions. Perchlorate ions are the only likely source of additional electrons in the acid and salt solutions. We use de-ionized water with greater than $18 \mathrm{M} \Omega / \mathrm{cm}$ resistance for the pure water samples, and obtain $2 M$ acid solutions by diluting reagent grade $70 \% \mathrm{HClO}_{4}$ (Sigma-Aldrich).

The $\sim 100$ fs excitation and probe pulses come from frequency conversion of the $800 \mathrm{~nm}$ light from an amplified Ti:sapphire laser consisting of an oscillator (Spectra Physics, Tsunami) and two consecutive multipass amplifiers. The laser system produces $1.6 \mathrm{~mJ}$ pulses with a $1 \mathrm{kHz}$ repetition rate, and we use up to $90 \%$ of the $800 \mathrm{~nm}$ light to generate excitation pulses. Frequency quadrupling the signal output of an optical parametric amplifier (Spectra Physics, OPA 800C) gives excitation pulses at $300 \mathrm{~nm}(4.13 \mathrm{eV})$, whereas frequency tripling or quadrupling the Ti:sapphire fundamental gives pulses at $267 \mathrm{~nm}(4.65 \mathrm{eV})$ and $200 \mathrm{~nm}(6.20 \mathrm{eV})$, respectively. Up to $3 \mu \mathrm{J}$ per pulse are incident on the sample in each case. A small fraction of the remaining $800 \mathrm{~nm}$ light passes onto a computer-controlled delay stage before we use it to produce probe pulses. Visible probe pulses come from generating white-light continuum in a water cell and passing it through an interference filter with a center wavelength of $650 \mathrm{~nm}$, while ultraviolet probe pulses come from frequency tripling the $800 \mathrm{~nm}$ light.

Two lenses separately focus the excitation and probe beams into a $100 \mu \mathrm{m}$ thick gear-pumped liquid jet, where they intersect at a small angle. Typical beam diameters at the sample are $30-100 \mu \mathrm{m}$ for the excitation beams and $15-30 \mu \mathrm{m}$ for the probe beams. Smaller beam diameters for $8.3 \mathrm{eV}$ excitation are necessary to compensate for the smaller two-photon absorption cross section compared with the higher excitation energies. The transient absorption signal at each probe wavelength changes quadratically with the intensity of the excitation pulses, confirming that the products come from two-photon excitation.

\section{RESULTS AND ANALYSIS}

\section{A. Two-photon excitation}

Figure 1 shows the transient absorption at probe wavelengths of 650 and $267 \mathrm{~nm}$ for all three excitation energies $(8.3,9.3$, and $12.4 \mathrm{eV})$. The insets show the same traces normalized over the range from 5 to $20 \mathrm{ps}$. In the case of the 8.3 and $9.3 \mathrm{eV}$ data, the beam diameter is approximately the same for both probe wavelengths, and a comparison of the relative absorption intensities, together with the absorption coefficients in Table I, ${ }^{36,37}$ provides a quantitative measure of the relative product yields from ionization and dissociation. For example, if ionization was the only channel the ratio of $\mathrm{OH}$ radicals and solvated electrons would be nearly equal and the absorption would be about 15 times stronger at $650 \mathrm{~nm}$ than $267 \mathrm{~nm}$. We find instead that $[\mathrm{OH}] /\left[e^{-}\right]$ $=3.3 \pm 1.0$ and $2.3 \pm 0.7$ for excitations at 8.3 and $9.3 \mathrm{eV}$, respectively, indicating that dissociation also plays a role. ${ }^{38} \mathrm{~A}$ similar comparison of the relative absorption strength at each probe wavelength is not possible for $12.4 \mathrm{eV}$ excitation because the probe beam diameters are not the same in that case.

Thomsen et $_{\text {al. }}{ }^{39}$ also measured the transient absorption following two-photon excitation at $9.3 \mathrm{eV}$ and they obtained 


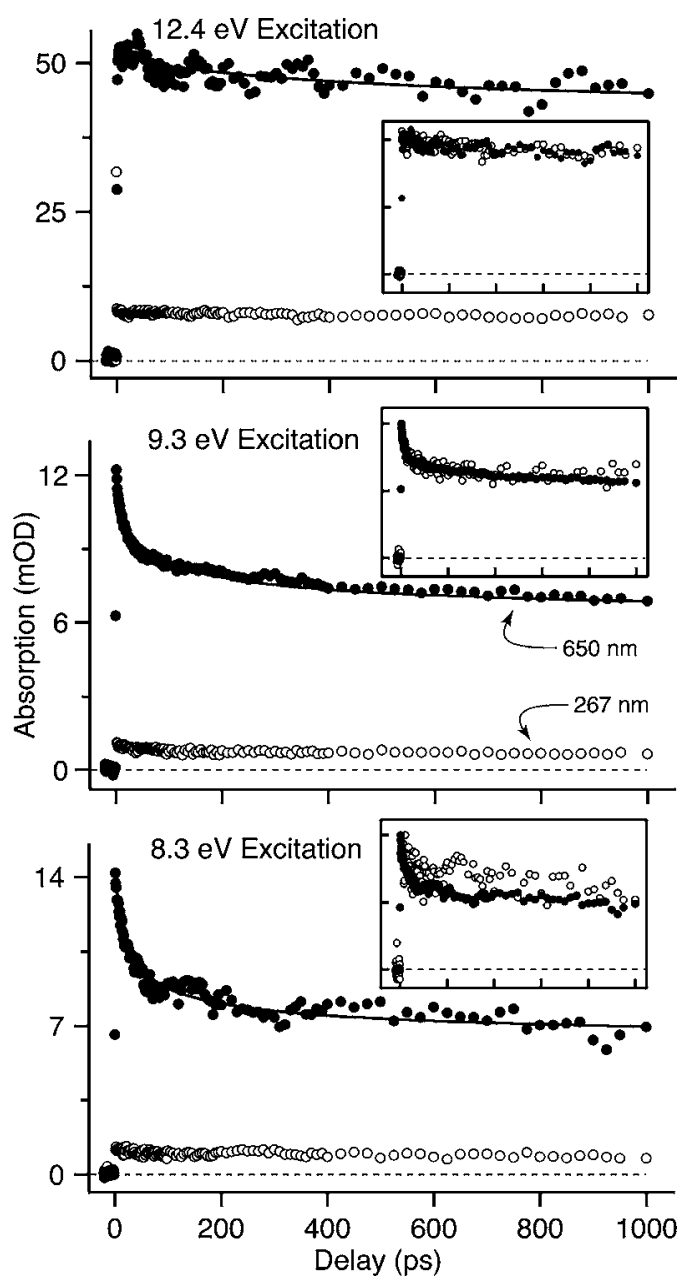

FIG. 1. Transient absorption following two-photon excitation at $12.4 \mathrm{eV}$ (top), $9.3 \mathrm{eV}$ (middle), and $8.3 \mathrm{eV}$ (bottom). The signal probed at $650 \mathrm{~nm}$ (closed circles) is entirely due to the absorption of light by solvated electrons, whereas the absorption at $267 \mathrm{~nm}$ (open circles) is due to both solvated electrons and $\mathrm{OH}$ radicals. The lines are best fits to the electron decay using the independent pairs model. The insets show the normalized traces.

$[\mathrm{OH}] /\left[e^{-}\right]=1.55$. Although their value is slightly smaller than ours, the two results are in reasonable agreement given the uncertainty of both measurements. The uncertainty primarily reflects variations in the spatial overlap of the probe and excitation beams. Slightly different overlaps for the two probe beams adversely affect the relative absorption measurement by causing the beams to sample different product concentration profiles.

An alternate method of determining the relative contribution to the absorption signal from each of the products is to compare the transient absorption in pure water and in an acid solution. ${ }^{40}$ Figure 2 compares the electron signal at

TABLE I. Product molar extinction coefficients $\left(M^{-1} \mathrm{~cm}^{-1}\right)$.

\begin{tabular}{ccc}
\hline \hline & $650 \mathrm{~nm}$ & $267 \mathrm{~nm}$ \\
\hline$e_{\mathrm{aq}}^{-}$ & $15500^{\mathrm{a}}$ & $600^{\mathrm{b}}$ \\
$\mathrm{OH}_{\mathrm{aq}}$ & 0 & $420^{\mathrm{b}}$ \\
\hline \hline
\end{tabular}

aㅏㅇㅡ Ref. 36.

${ }^{b}$ From Ref. 37. Although other estimates of the absorption coefficients at $267 \mathrm{~nm}$ vary by as much as $\pm 15 \%$, the difference does not have a significant impact on our results.

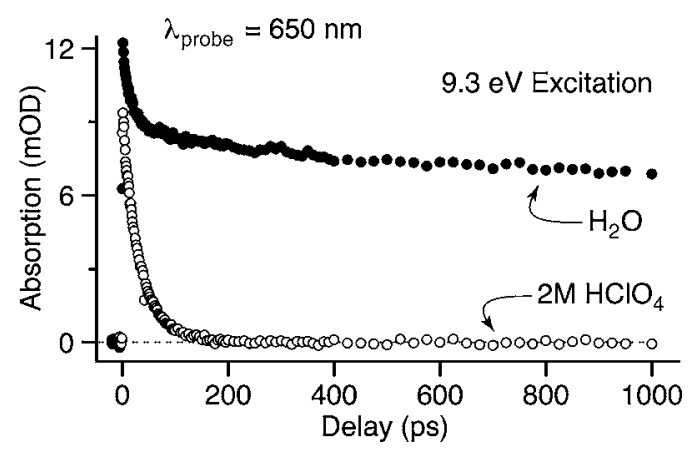

FIG. 2. Transient absorption at $650 \mathrm{~nm}$ following $9.3 \mathrm{eV}$ excitation of pure water (closed circles) and $2 \mathrm{M} \mathrm{HClO}_{4}$ solution (open circles). The electron signal decays to the baseline for the acid solution due to reaction of electrons with excess protons.

$650 \mathrm{~nm}$ for pure water (closed circles) and for a $2 M$ solution of $\mathrm{HClO}_{4}$ (open circles) following $9.3 \mathrm{eV}$ excitation. The ionization of pure water results in the usual geminate recombination kinetics, but the electron signal exponentially decays to the baseline within about $100 \mathrm{ps}$ in the acid solution due to bimolecular reaction with excess protons $\left(k_{3}=1.2\right.$ $\times 10^{10} M^{-1} \mathrm{~s}^{-1}$ for a $2 \mathrm{M}$ solution of $\left.\mathrm{HClO}_{4}\right)^{41}$

$$
e_{(\mathrm{aq})}^{-}+\mathrm{H}_{3} \mathrm{O}_{(\mathrm{aq})}^{+} \rightarrow \mathrm{H}_{(\mathrm{aq})}+\mathrm{H}_{2} \mathrm{O}_{(l)} .
$$

The solvated electron signal also decays to the baseline within about $100 \mathrm{ps}$ for 8.3 and $12.4 \mathrm{eV}$ excitations of the acid solution (not shown).

The data in Fig. 3 are the corresponding transient absorption traces at a probe wavelength of $267 \mathrm{~nm}$ for excitation of pure water and $2 M$ acid solution. The ultraviolet absorption decays more in the acid solution than in pure water following excitations at 12.4 and $9.3 \mathrm{eV}$, but the signal is about the same in each for $8.3 \mathrm{eV}$ excitation. Because $\mathrm{H}_{3} \mathrm{O}^{+}$ ions and $\mathrm{H}$ atoms do not absorb at either 650 or $267 \mathrm{~nm}$, the primary difference between the traces in pure water and in the acid solution is the additional decay of electrons in the latter. ${ }^{42}$ In the top panel of the figure the ultraviolet absorption at long delay times is about $57 \%$ weaker for the acid solution relative to pure water, indicating that electrons account for about $57 \%$ of the transient signal following $12.4 \mathrm{eV}$ excitation. Based on the absorption cross sections of the products (Table I), the $57 \%$ contribution of electrons to the $267 \mathrm{~nm}$ signal gives $[\mathrm{OH}] /\left[e^{-}\right]=1.1 \pm 0.3$. Similarly, for $9.3 \mathrm{eV}$ excitation, the electron contributes about $45 \%$ of the absorption signal, and the resulting value $[\mathrm{OH}] /\left[e^{-}\right]$ $=1.7 \pm 0.9$ is in good agreement with the ratio from the transient absorption data for pure water.

In contrast with the results at 9.3 and $12.4 \mathrm{eV}$, the similarity of the traces in the bottom panel of Fig. 3 indicates that electrons do not make a significant contribution to the transient ultraviolet absorption for $8.3 \mathrm{eV}$ excitation. Considering the signal-to-noise ratio in our experiment, we estimate that electrons contribute less than $15 \%$ of the $267 \mathrm{~nm}$ absorption signal. Although the resulting ratio $[\mathrm{OH}] /\left[e^{-}\right]>8$ is somewhat higher than we obtain from the relative absorption measurement in Fig. 1, we believe that the acid quenching experiment gives the more accurate estimate in light of the relatively large uncertainty in comparing the absorption 

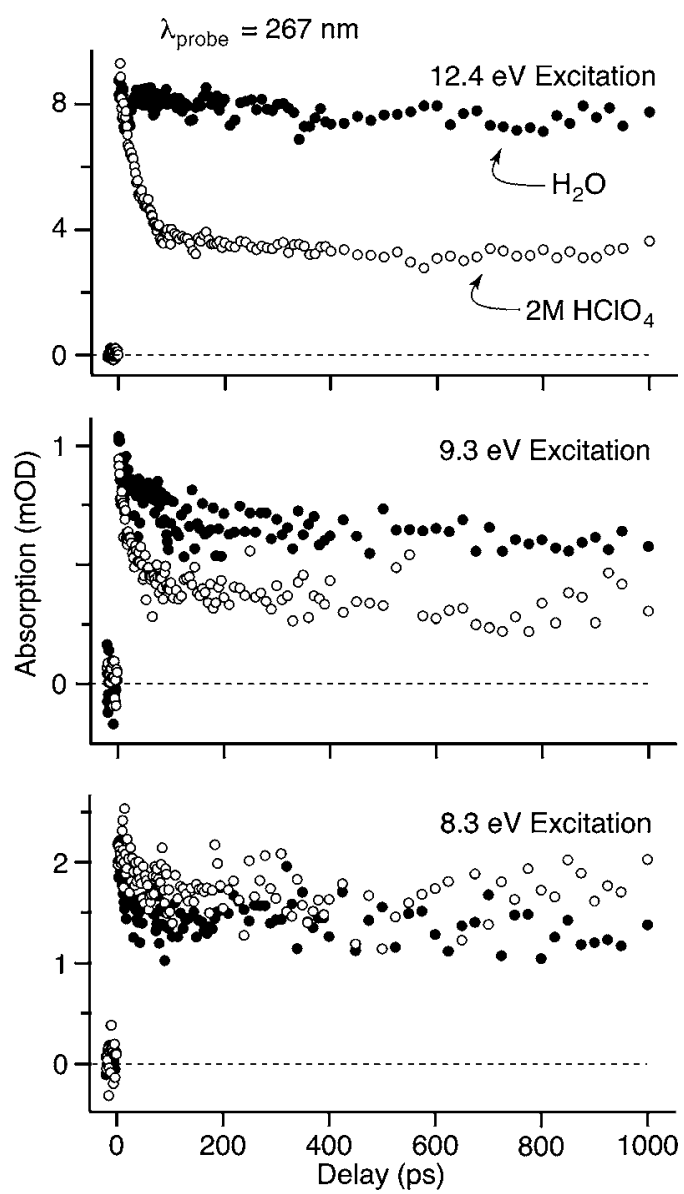

FIG. 3. Transient absorption at $267 \mathrm{~nm}$ following excitation of pure water (closed circles) and $2 \mathrm{M} \mathrm{HClO}_{4}$ solution (open circles). The signal in the top and middle panels decays more in the acid solution than in pure water, indicating the contribution of solvated electrons to the absorption. The signal in the bottom panel does not decay in the acid solution relative to pure water because the contribution from solvated electrons is very small at that excitation energy.

strength at two probe wavelengths. The acid quenching experiment is independent of the relative overlap of excitation and probe beams at different wavelengths because that measurement compares the transient data for pure water and acid solutions at a single wavelength.

The $<15 \%$ electron contribution implies that $\mathrm{OH}$ radicals from the dissociation channel are responsible for more than $75 \%$ of the signal. We conclude that $\mathrm{OH}$ radicals from dissociation are the dominant absorbing species probed at $267 \mathrm{~nm}$ following $8.3 \mathrm{eV}$ excitation, and that the dissociation yield is several times larger than the ionization yield at that energy. The dissociation yields are summarized in Table II. Consistent with the large contribution of solvated electrons to the ultraviolet absorption signal for 9.3 and $12.4 \mathrm{eV}$ excitations, the normalized traces in the insets of Fig. 1 decay at similar rates for each probe wavelength. In contrast, the decay is noticeably different for the normalized traces in the bottom panel of Fig. 1, where the different decays for each probe wavelength are a result of probing the products from different reactions; the solvated electron signal in the visible reveals the geminate recombination kinetics for the minor ionization channel, while recombining $\mathrm{OH}$ radicals formed via the dissociation channel dominate the signal in the ultraviolet.
TABLE II. Ionization and dissociation yields for two-photon excitation.

\begin{tabular}{cccc}
\hline \hline Energy $(\mathrm{eV})$ & Ionization yield $(\%)$ & $\left(\mathrm{OH}_{\mathrm{aq}}\right) /\left[e_{\mathrm{aq}}^{-}\right]^{\mathrm{a}}$ & Dissociation yield $^{\mathrm{b}}(\%)$ \\
\hline 12.4 & $\sim 100^{\mathrm{c}}$ & $1.1 \pm 0.3$ & $<10$ \\
9.3 & $44^{\mathrm{d}}$ & $1.7 \pm 0.9$ & 31 \\
8.3 & $12^{\mathrm{d}}$ & $>8$ & $>84$ \\
\hline
\end{tabular}

${ }^{\mathrm{a}}$ From the acid quenching experiments in this work

${ }^{\mathrm{b}}$ Approximate dissociation yield assuming constant $\left[\mathrm{OH}_{\mathrm{aq}}\right] /\left[e_{\mathrm{aq}}^{-}\right]$.

${ }^{\mathrm{c}}$ Reference 33.

${ }^{\mathrm{d}}$ From Ref. 32, extrapolated to $t=0$ ps using the independent pairs model and the values of $\left\langle r_{0}\right\rangle$ from the data in this work.

\section{B. Models of geminate recombination}

Solvated electrons are the only species that absorb light at $650 \mathrm{~nm}$, therefore the decay of the absorption signal at that wavelength reflects the recombination of electrons with their geminate partners following the two-photon ionization of water.

$$
\begin{aligned}
& e_{(\mathrm{aq})}^{-}+\mathrm{OH}_{(\mathrm{aq})} \rightarrow \mathrm{OH}_{(\mathrm{aq})}^{-}, \\
& e_{(\mathrm{aq})}^{-}+\mathrm{H}_{3} \mathrm{O}_{(\mathrm{aq})}^{+} \rightarrow \mathrm{H}_{(\mathrm{aq})}+\mathrm{H}_{2} \mathrm{O}_{(l)} .
\end{aligned}
$$

We fit the electron signal at this probe wavelength using the independent pairs model, which assumes that the competing recombination reactions (4) and (5) proceed independently. ${ }^{43,44}$ The independent pairs model accurately reproduces the signal decay using a single variable fit parameter $\left\langle r_{0}\right\rangle$, which is the average initial separation of solvated electrons and their ionization counterparts in thermal equilibrium with the solvent. ${ }^{26}$ We assume a Gaussian distribution of electron ejection lengths, centered at the origin of the ionization site, with the value of $\left\langle r_{0}\right\rangle$ related to the width of the distribution. Larger initial separations lead to less recombination because the geminate species are less likely to diffusively encounter each other. Fits to the $650 \mathrm{~nm}$ electron decay data using the independent pairs model (solid lines in Fig. 1) give average ejection lengths of $\left\langle r_{0}\right\rangle=1.0 \pm 0.2$, $1.4 \pm 0.2$, and $3.8 \pm 0.5 \mathrm{~nm}$ for $8.3,9.3$, and $12.4 \mathrm{eV}$ excitations, respectively. These values are in excellent agreement with our previous work studying in detail the variation of the ejection length with excitation energy in the range from 7.8 to $12.4 \mathrm{eV}^{25,26}$

Figure 4 shows the transient decay of the $267 \mathrm{~nm}$ ab-

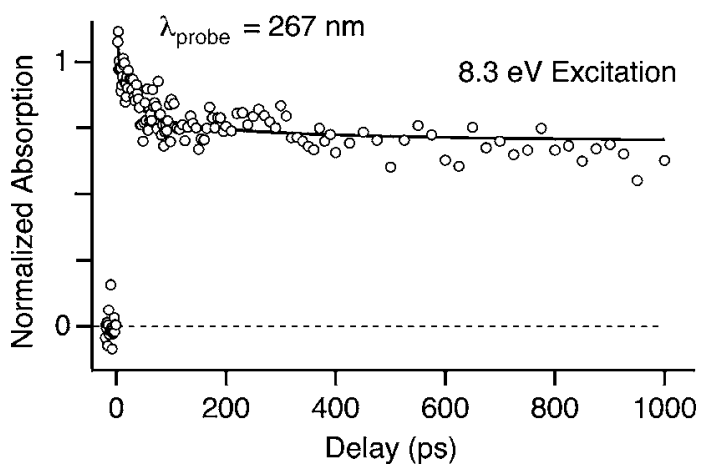

FIG. 4. Transient $267 \mathrm{~nm}$ absorption in pure water following two-photon excitation at $8.3 \mathrm{eV}$. The line is a best fit to the data using the simple geminate recombination model from the text. 
sorption signal following $8.3 \mathrm{eV}$ excitation, where $\mathrm{OH}$ radicals from the dissociation of water are largely responsible for the kinetics. We ignore the small contribution to the signal from the ionization channel and fit the data with a simple model for diffusion-limited recombination of the dissociation products.

$$
\mathrm{H}_{(\mathrm{aq})}+\mathrm{OH}_{(\mathrm{aq})} \rightarrow \mathrm{H}_{2} \mathrm{O}_{(l)}
$$

The recombination model gives a time-dependent expression for the survival probability of $\mathrm{OH}$ radicals as follows:

$$
[\mathrm{OH}](t)=[\mathrm{OH}](0) \cdot\left[1-\left(\frac{R}{r_{\mathrm{H}-\mathrm{OH}}}\right) \cdot \operatorname{erfc}\left(\frac{r_{\mathrm{H}-\mathrm{OH}}-R}{\sqrt{4 D t}}\right)\right],
$$

where $r_{\mathrm{H}-\mathrm{OH}}$ is the initial separation of a pair of fragments, $D$ is the sum of their diffusion rates, and $R$ is the reaction radius, within which the species recombine with unit yield. ${ }^{45}$ The steady-state reaction rate constant $\left(k_{6}=4 \pi N_{A} R D=2.0\right.$ $\left.\times 10^{10} M^{-1} \mathrm{~s}^{-1}\right)$ and joint diffusion constant $\left(D=D_{\mathrm{H}}+D_{\mathrm{OH}}\right.$ $=9.8 \mathrm{~nm}^{2} / \mathrm{ns}$ ) give a reaction radius of $R=0.27 \mathrm{~nm}$, assuming diffusion-limited reaction ${ }^{46}\left(N_{A}\right.$ is Avogadro's number).

The solid line in the figure is a fit to the data using numerical integration to account for a distribution of fragment separation lengths. A Gaussian distribution centered away from the origin (i.e., $r \neq 0$ ) has been used to model similar dissociation reactions. ${ }^{47}$ The best fit to the data gives an average separation length of $\left\langle r_{\mathrm{H}-\mathrm{OH}}\right\rangle=0.7 \pm 0.2 \mathrm{~nm}$, regardless of whether we use a Gaussian function or a delta function distribution. Even using a Gaussian function centered at the origin, similar to the form of the electron distribution in the independent pairs model for ionization described above, gives the same value for the average separation of $\mathrm{H}$ and $\mathrm{OH}$. A source of uncertainty in this analysis is the small contribution to the signal from the ionization products. Ignoring the contribution from ionization leads us to overestimate the recombination yield for the dissociation products because the ionization products are more likely to recombine than the dissociation products (see the inset in the bottom panel of Fig. 1). Therefore, the $r_{\mathrm{H}-\mathrm{OH}}$ separation length determined in our analysis is likely a lower bound.

\section{DISCUSSION}

\section{A. Dissociation dynamics}

An important result from our measurement is the large separation of $\mathrm{H}$ and $\mathrm{OH}$ fragments following the dissociation of a water molecule in the liquid $\left(\left\langle r_{\mathrm{H}-\mathrm{OH}}\right\rangle=0.7 \pm 0.2 \mathrm{~nm}\right)$. Dissociating $\mathrm{H}$ atoms escape the solvent cage and one or two additional solvent shells, suggesting that they are produced with significant initial kinetic energy and that hydrogen bonding does not drastically change the direct dissociation channel of a water molecule in the liquid relative to the gas phase. A recent molecular dynamics simulation of dissociating $\mathrm{H}_{2} \mathrm{O}$ in crystalline and amorphous ice supports this interpretation. ${ }^{34,35}$ The simulation treats the nuclear dynamics classically using an $a b$ initio potential energy surface for isolated water to describe the intramolecular dynamics of the dissociating molecule, a modified TIP3P model to describe its interaction with the solvent, and the TIP4P model for the intermolecular interaction of bulk water molecules. By neglecting the effects of hydrogen bonding on the excited state dynamics, the simulation provides a reference point in which cooperative effects of the liquid do not significantly alter the dissociation dynamics relative to the gas phase. Our experimental result is similar to the outcome of the simulation, where $\mathrm{H}$ atoms travel an average distance of $0.8 \mathrm{~nm}$ prior to thermalization. ${ }^{48}$ That similarity and the large separation length that we measure suggest that hydrogen bonding effects do not inhibit direct dissociation in the liquid.

On the other hand, recent high-level ab initio calculations of the first excited state of the dimer ${ }^{19-21}$ and other small water clusters ${ }^{20}$ suggest that hydrogen bonding has a strong influence on the shape of the potential energy surface. The calculations reveal a low barrier to dissociation along the $\mathrm{O}-\mathrm{H}$ stretch coordinate of the hydrogen bond donor that does not exist in the isolated molecule. In one limit, such a barrier would inhibit motion along the $\mathrm{O}-\mathrm{H}$ stretch, thereby dissipating excess energy from the dissociating fragments and limiting the ability of the $\mathrm{H}$ atom to penetrate the solvent. By inhibiting the direct dissociation channel, a barrier also potentially increases the lifetime of the excited state and reduces the quantum yield for dissociation. ${ }^{20}$ The time scale of roughly $1 \mathrm{ps}$ for hydrogen bond breaking in the electronic ground state ${ }^{5,6}$ would give an upper limit for the excited state lifetime that is long enough for an alternate decay path to compete efficiently with direct dissociation. In the absence of a barrier, dissociation occurs on the time scale of an $\mathrm{O}-\mathrm{H}$ stretch vibration $(\sim 10 \mathrm{fs})$.

The excitation energy determines the impact of such a barrier on the excited state dynamics. At low excitation energies the barrier may be insurmountable and have a profound influence on the dynamics, but at energies sufficiently high to overcome the barrier it has little effect. Indirect measurements of the dissociation yield for one-photon excitation of liquid water reflect this energy dependence. Dissociation occurs with only $45 \%$ yield for one-photon excitation at $6.7 \mathrm{eV},{ }^{29}$ indicating that a competing decay channel capable of relaxing the molecule to the ground state is important at that energy, whereas the yield increases to $70 \%$ at $8.4 \mathrm{eV} .^{28}$ The dissociation yield increases with energy across this range as the influence of the barrier decreases.

Our measurement of the relative yields of ionization and dissociation for two-photon excitation (see Table II) also indicates a dominant dissociation channel at $8.3 \mathrm{eV}$. However, it is important to note that the character of the excited state produced with the same total excitation energy may be different for one- and two-photon transitions because of the different selection rules. ${ }^{49}$ Even though $8.3 \mathrm{eV}$ is at the center of the first absorption band in the one-photon spectrum of the liquid, a two-photon excitation at this energy may also excite into the tail of higher-lying excited states. Experiments to better understand the relative two-photon cross sections are ongoing in our laboratories but the similar one- and two-photon dissociation yields suggest that there is not a significant difference in the excited state for excitation at $8.3 \mathrm{eV}$. If indeed we prepare predominantly the first excited 
state in our experiment, the large dissociation yield implies that the barrier is relatively inefficient at that energy. A large dissociation yield is consistent with the production of $\mathrm{H}$ atoms with substantial kinetic energy because a barrier does not inhibit the dissociation channel. Above $8.3 \mathrm{eV}$, the dissociation yield decreases as ionization becomes increasingly important, ${ }^{33}$ likely due to the changing character of the excited state. There are no theoretical calculations in the literature that explore the effect of hydrogen bonding on the dissociation coordinate for higher excited states of water, although these would clearly be desirable.

It is interesting to consider the role of a barrier to dissociation in the context of the hydrogen bonding structure of liquid water. ${ }^{50-53}$ The dissociation yield may provide a measure of the extent of hydrogen bonding in the liquid if molecules in various solvent geometries experience different barrier heights and therefore different dynamics. In this picture, the solvent environment controls the dissociation yield because the height of the barrier depends on the hydrogen bonding structure of the liquid. For instance, weak hydrogen bond donors may experience a small barrier that does little to inhibit dissociation, whereas strongly bound species in tetrahedral, icelike configurations (with longer-lived excited states $^{54}$ ) may dissociate indirectly or relax through a different mechanism. Calculations by Chipman ${ }^{21}$ indicate that nonbonded or weakly hydrogen bonded molecules preferentially dissociate because dissociation along a nonhydrogen bonded $\mathrm{O}-\mathrm{H}$ stretch is the lowest energy path in the first excited state of the dimer. If fully coordinated waters preferentially decay through an alternate mechanism (e.g., the quantum yield of dissociation in crystalline ice is less than unity), then the branching among channels depends on the extent of hydrogen bonding in the liquid. Temperature-dependent measurements of the dissociation yield, particularly at low excitation energies, would be informative, because raising the temperature decreases hydrogen bonding in the liquid and therefore reduces its influence on the dynamics. ${ }^{55}$

\section{B. Ionization mechanism}

Previous studies of ionization in liquid water reveal that the mechanism depends on the excitation energy. ${ }^{22-26}$ Direct ionization to produce $\mathrm{H}_{2} \mathrm{O}_{(\mathrm{aq})}^{+}$and a quasifree electron is only possible for excitations above the energy for vertical electron ejection at about $11 \mathrm{eV} .^{26}$ For lower excitation energies the excited molecule and its environment must reorganize in order for the system to attain a favorable geometry for electron ejection because vertical transitions to a continuum electron state are increasingly unlikely. We restrict the current discussion to excitation energies below $9.5 \mathrm{eV}$, where nuclear motion plays a central role in the ionization mechanism. ${ }^{32}$

The prevailing theory for ionization in this energy regime is an excited state proton-coupled electron transfer mechanism, in which electron ejection and nuclear motion along the proton transfer coordinate are simultaneous. ${ }^{32} \mathrm{Al}$ though the exact details of this mechanism are somewhat vague, it may explain many of the experimental properties of ionization, such as the nearly constant electron ejection length $\left\langle r_{0}\right\rangle$ below $9 \mathrm{eV}$ and the exponential increase of the ionization yield with energy. The ejection length is independent of the excitation energy because that length is determined by the location of electron trap states, which does not change with energy. On the other hand, more traps are available at higher energies and the ionization yield increases accordingly. A proton-coupled electron transfer mechanism implies that ionization competes with dissociation, and that the nature of the excited state determines their relative yield.

Another proposed mechanism for low energy excitation is one in which translationally hot $\mathrm{H}$ atoms from dissociation collide and react with a neighboring water molecule to produce an electron and a hydronium ion. ${ }^{25}$

$$
\mathrm{H}+\mathrm{H}_{2} \mathrm{O}_{(l)} \rightarrow \mathrm{H}_{3} \mathrm{O}_{(\mathrm{aq})}^{+}+e_{(\mathrm{aq})}^{-}
$$

Only sufficiently energetic $\mathrm{H}$ atoms react via this mechanism, which has an activation energy of about $0.7 \mathrm{eV}{ }^{56} \mathrm{An}$ upper limit to the total available energy for the dissociating fragments is about $3.2 \mathrm{eV}$ using the gas phase bond dissociation energy of $5.1 \mathrm{eV}$ and the $8.3 \mathrm{eV}$ excitation energy. ${ }^{57}$ Although hydrogen bonding may alter the disposal of energy for the dissociation reaction in solution, $88 \%$ of the excess energy goes into translation of the $\mathrm{H}$ atom in the gas phase. ${ }^{3}$ Thus, the reaction of $\mathrm{H}$ atoms with a neighboring water molecule is indeed energetically feasible if the liquid environment does not have a dramatic influence on the dissociation dynamics.

In this picture, dissociation plays an essential role in ionization, rather than being a competing process, and the efficiency of reaction (8) determines the relative branching. The increasing likelihood of $\mathrm{H}$ atoms having enough energy to overcome the barrier to reaction would explain the exponential increase of the ionization yield with energy. On the other hand, the location of electron trap sites in the liquid once again determines the electron ejection length, and is independent of the excitation energy.

Other reactions involving $\mathrm{H}$ atoms from dissociation potentially play a role as well. Two such reactions with comparable activation energies are hydrogen abstraction $(0.7 \mathrm{eV}$ activation energy $\left.\mathrm{y}^{58}\right)$ and hydrogen exchange $(0.9 \mathrm{eV}$ barrier in the gas phase). ${ }^{59}$

$$
\begin{aligned}
& \mathrm{H}+\mathrm{H}_{2} \mathrm{O}_{(l)} \rightarrow \mathrm{H}_{2(\mathrm{aq})}+\mathrm{OH}_{(\mathrm{aq})}, \\
& \mathrm{H}^{\prime}+\mathrm{H}_{2} \mathrm{O}_{(l)} \rightarrow \mathrm{H}^{\prime} \mathrm{OH}_{(l)}+\mathrm{H}_{(\mathrm{aq})} .
\end{aligned}
$$

In the limit of ballistic $\mathrm{H}$ atoms, the efficiencies of reactions (8)-(10) depend on the collision energy and the impact angle of the reactants. Only the first collision or two is likely to have enough energy to overcome the reaction barrier, even for a favorable impact parameter, because collisions rapidly dissipate excess energy from the $\mathrm{H}$ atom. ${ }^{60}$ The approximately tetrahedral arrangement of liquid water preferentially aligns the dissociating $\mathrm{O}-\mathrm{H}$ bond toward the $\mathrm{O}$ atom on the nearest neighboring water molecule. Thus, the most likely first collision has an unfavorable orientation for hydrogen abstraction that limits the yield of reaction (9). ${ }^{59}$ The transition state geometry of reaction (10), on the other hand, is a trigonal $\mathrm{H}_{3} \mathrm{O}$ molecule that is readily accessible by dissociating a water molecule in a relaxed configuration of the 
liquid. ${ }^{59}$ Although speculative, it is interesting to consider that the $\mathrm{H}_{3} \mathrm{O}$ species could also be an intermediate state for ionization via reaction $(8) .{ }^{18}$

The excited state potential energy surface plays an important role in both of the proposed mechanisms of ionization. The essential difference between them is the role of hydrogen bonding in the excited state dynamics. In one limit, hydrogen bonding promotes excited state proton transfer, whereas in the other limit prompt dissociation similar to the gas phase produces translationally hot $\mathrm{H}$ atoms that react with a neighboring water molecule. Both mechanisms explain the constant ejection length and exponentially increasing ionization yield below $9 \mathrm{eV}$. Although we cannot exclude the possible role of proton-coupled electron transfer, our observation of prompt dissociation favors the hot $\mathrm{H}$ atom reaction mechanism of ionization.

\section{SUMMARY}

Transient absorption spectroscopy reveals the relative yields of ionization and dissociation following the twophoton excitation of liquid water at $8.3,9.3$, and $12.4 \mathrm{eV}$. The ionization channel dominates for the highest excitation energy and the two decay channels occur with nearly equal probability for $9.3 \mathrm{eV}$ excitation. On the other hand, the dissociation channel dominates for $8.3 \mathrm{eV}$ excitation, where the transient decay of the $267 \mathrm{~nm}$ absorption signal provides information about the dissociation reaction. We find from the geminate kinetics of the $\mathrm{OH}$ radical that the average initial separation of $\mathrm{H}$ and $\mathrm{OH}$ dissociation fragments is $0.7 \pm 0.2 \mathrm{~nm}$, about two solvent shells. This first determination of the dynamics occurring in the dissociation channel provides a new insight into the overall excited state relaxation of liquid water.

The large fragment separation that we observe implies that dissociation produces $\mathrm{H}$ atoms with significant initial kinetic energy. Our measurement points to a direct dissociation channel that is not significantly inhibited by a barrier on the excited state potential energy surface even though onephoton dissociation yields suggest that a barrier may play a role at lower excitation energies. The production of ballistic $\mathrm{H}$ atoms from dissociation tenuously supports a picture of the ionization mechanism in which $\mathrm{H}$ atoms react with neighboring water molecules to give the ionization products, although we cannot rule out a proton-coupled electron transfer mechanism. The hot $\mathrm{H}$ atom reaction mechanism is an appealing picture considering that direct dissociation of isolated water molecules produces $\mathrm{H}$ atoms with significant kinetic energy.

\section{ACKNOWLEDGMENTS}

The authors wish to thank C. D. Jonah for several helpful conversations. This work was performed at Argonne National Laboratory and was supported by the U.S. Department of Energy, Office of Science, Office of Basic Energy Sciences, under Contract No. DE-AC02-06CH11357. One of the authors (S.E.B.) is supported by the National Science Foundation (CHE 0311814 and 0617060).
${ }^{1}$ B. C. Garrett, D. A. Dixon, D. M. Camaioni, et al., Chem. Rev. (Washington, D.C.) 105, 355 (2005).

${ }^{2}$ V. Engel, V. Staemmler, R. L. Vanderwal, F. F. Crim, R. J. Sension, B. Hudson, P. Andresen, S. Hennig, K. Weide, and R. Schinke, J. Phys. Chem. 96, 3201 (1992).

${ }^{3}$ P. Andresen, G. S. Ondrey, B. Titze, and E. W. Rothe, J. Chem. Phys. 80, 2548 (1984)

${ }^{4}$ J. M. Heller, Jr., R. N. Hamm, R. D. Birkhoff, and L. R. Painter, J. Chem. Phys. 60, 3483 (1974).

${ }^{5}$ C. J. Fecko, J. D. Eaves, J. J. Loparo, A. Tokmakoff, and P. L. Geissler, Science 301, 1698 (2003).

${ }^{6}$ J. B. Asbury, T. Steinel, C. Stromberg, S. A. Corcelli, C. P. Lawrence, J. L. Skinner, and M. D. Fayer, J. Phys. Chem. A 108, 1107 (2004).

${ }^{7}$ M. L. Cowan, B. D. Bruner, N. Huse, J. R. Dwyer, B. Chugh, E. T. J. Nibbering, T. Elsaesser, and R. J. D. Miller, Nature (London) 434, 199 (2005).

${ }^{8}$ B. Brena, D. Nordlund, M. Odelius, H. Ogasawara, A. Nilsson, and L. G. M. Pettersson, Phys. Rev. Lett. 93, 148302 (2004).

${ }^{9}$ M. Odelius, H. Ogasawara, D. Nordlund, et al., Phys. Rev. Lett. 94, 227401 (2005).

${ }^{10}$ B. D. Bursulaya and H. J. Kim, J. Chem. Phys. 109, 4911 (1998).

${ }^{11}$ B. D. Bursulaya, J. Jeon, C. N. Yang, and H. J. Kim, J. Phys. Chem. A 104, 45 (2000).

${ }^{12}$ O. Christiansen, T. M. Nymand, and K. V. Mikkelsen, J. Chem. Phys. 113, 8101 (2000).

${ }^{13}$ P. H. Hahn, W. G. Schmidt, K. Seino, M. Preuss, F. Bechstedt, and J. Bernholc, Phys. Rev. Lett. 94, 037404 (2005).

${ }^{14}$ A. Osted, J. Kongsted, K. V. Mikkelsen, and O. Christiansen, J. Phys. Chem. A 108, 8646 (2004).

${ }^{15}$ M. Aschi, M. D'Abramo, C. Di Teodoro, A. Di Nola, and A. Amadei, ChemPhysChem 6, 53 (2005).

${ }^{16}$ R. M. Sosa, P. Gardiol, and O. N. Ventura, J. Mol. Struct. 297, 337 (1993).

${ }^{17}$ J. N. Harvey, J. O. Jung, and R. B. Gerber, J. Chem. Phys. 109, 8747 (1998).

${ }^{18}$ A. L. Sobolewski and W. Domcke, J. Chem. Phys. 122, 184320 (2005).

${ }^{19}$ L. Valenzano, M. C. van Hemert, and G. J. Kroes, J. Chem. Phys. 123, 034303 (2005).

${ }^{20}$ D. M. Chipman, J. Chem. Phys. 122, 044111 (2005).

${ }^{21}$ D. M. Chipman, J. Chem. Phys. 124, 044305 (2006).

${ }^{22}$ M. U. Sander, K. Luther, and J. Troe, J. Phys. Chem. 97, 11489 (1993).

${ }^{23}$ M. U. Sander, M. S. Gudiksen, K. Luther, and J. Troe, Chem. Phys. 258, 257 (2000).

${ }^{24}$ A. Bernas, C. Ferradini, and J. P. Jay-Gerin, Chem. Phys. 222, 151 (1997).

${ }^{25}$ R. A. Crowell and D. M. Bartels, J. Phys. Chem. 100, 17940 (1996).

${ }^{26}$ C. G. Elles, A. E. Jailaubekov, R. A. Crowell, and S. E. Bradforth, J. Chem. Phys. 125, 044515 (2006).

${ }^{27}$ J. Barrett and J. H. Baxendale, Trans. Faraday Soc. 56, 37 (1960).

${ }^{28}$ U. Sokolov and G. Stein, J. Chem. Phys. 44, 2189 (1966).

${ }^{29}$ U. Sokolov and G. Stein, J. Chem. Phys. 44, 3329 (1966).

${ }^{30}$ J. W. Boyle, J. A. Ghormley, C. J. Hochanadel, and J. F. Riley, J. Phys. Chem. 73, 2886 (1969).

${ }^{31}$ D. N. Nikogosyan, A. A. Oraevsky, and V. I. Rupasov, Chem. Phys. 77, 131 (1983).

${ }^{32}$ D. M. Bartels and R. A. Crowell, J. Phys. Chem. A 104, 3349 (2000).

${ }^{33}$ A. Mozumder, Phys. Chem. Chem. Phys. 4, 1451 (2002).

${ }^{34}$ S. Andersson, G. J. Kroes, and E. F. van Dishoeck, Chem. Phys. Lett. 408, 415 (2005).

${ }^{35}$ S. Andersson, A. Al-Halabi, G. J. Kroes, and E. F. van Dishoeck, J. Chem. Phys. 124, 064715 (2006).

${ }^{36}$ F.-Y. Jou and G. R. Freeman, J. Phys. Chem. 83, 2383 (1979).

${ }^{37}$ S. O. Nielsen, B. D. Michael, and E. J. Hart, J. Phys. Chem. 80, 2482 (1976).

${ }^{38} \mathrm{~A}$ slight excess of $\mathrm{OH}$ radicals over electrons is expected even for pure ionization, because $20 \%$ of recombining electrons react with the geminate $\mathrm{H}_{3} \mathrm{O}^{+}$ion rather than the $\mathrm{OH}$ radical, but the effect of the competing recombination reactions following ionization is too small to account for the large excess of $\mathrm{OH}$ radicals that we observe.

${ }^{39}$ C. L. Thomsen, D. Madsen, S. R. Keiding, J. Thogersen, and O. Christiansen, J. Chem. Phys. 110, 3453 (1999).

${ }^{40}$ C. D. Jonah and J. R. Miller, J. Phys. Chem. 81, 1974 (1977).

${ }^{41}$ C. D. Jonah, J. R. Miller, and M. S. Matheson, J. Phys. Chem. 81, 931 (1977). 
${ }^{42}$ The decay of the $\mathrm{OH}$ radical is not affected by the conversion of electrons into $\mathrm{H}$ atoms in the acid solution because both species react with $\mathrm{OH}$ radicals at a similar rate (see Ref. 40).

${ }^{43}$ S. M. Pimblott, J. Phys. Chem. 95, 6946 (1991).

${ }^{44}$ T. Goulet and J. P. Jay-Gerin, J. Chem. Phys. 96, 5076 (1992).

${ }^{45}$ C. G. Elles and F. F. Crim, Annu. Rev. Phys. Chem. 57, 273 (2006).

${ }^{46}$ N. J. B. Green, M. J. Pilling, S. M. Pimblott, and P. Clifford, J. Phys. Chem. 94, 251 (1990).

${ }^{47}$ C. L. Thomsen, D. Madsen, J. A. Poulsen, J. Thogersen, S. J. K. Jensen, and S. R. Keiding, J. Chem. Phys. 115, 9361 (2001).

${ }^{48}$ The simulation also reveals a tail in the distribution of separation lengths that extends to much longer distances, but the fits to our data are not sensitive enough to reveal such an asymmetry.

${ }^{49}$ M. J. Paterson, J. Kongsted, O. Christiansen, K. V. Mikkelsen, and C. B. Nielsen, J. Chem. Phys. 125, 184501 (2006).

${ }^{50}$ P. Wernet, D. Nordlund, U. Bergmann, et al., Science 304, 995 (2004).

${ }^{51}$ J. D. Smith, C. D. Cappa, K. R. Wilson, B. M. Messer, R. C. Cohen, and
R. J. Saykally, Science 306, 851 (2004).

${ }^{52}$ J. D. Eaves, J. J. Loparo, C. J. Fecko, S. T. Roberts, A. Tokmakoff, and P. L. Geissler, Proc. Natl. Acad. Sci. U.S.A. 102, 13019 (2005).

${ }^{53}$ R. L. C. Wang, H. J. Kreuzer, and M. Grunze, Phys. Chem. Chem. Phys. 8, 4744 (2006).

${ }^{54}$ N. G. Petrik and G. A. Kimmel, Phys. Rev. Lett. 90, 166102 (2003).

${ }^{55}$ A. J. Lock and H. J. Bakker, J. Chem. Phys. 117, 1708 (2002).

${ }^{56}$ H. Shiraishi, G. R. Sunaryo, and K. Ishigure, J. Phys. Chem. 98, 5164 (1994).

${ }^{57}$ P. Maksyutenko, T. R. Rizzo, and O. V. Boyarkin, J. Chem. Phys. 125, 181101 (2006).

${ }^{58}$ D. Swiatla-Wojcik and G. V. Buxton, Radiat. Phys. Chem. 74, 210 (2005).

${ }^{59}$ I. W. M. Smith and F. F. Crim, Phys. Chem. Chem. Phys. 4, 3543 (2002).

${ }^{60}$ A. Yabushita, D. Kanda, N. Kawanaka, M. Kawasaki, and M. N. R. Ashfold, J. Chem. Phys. 125, 133406 (2006). 